\title{
A UAV-BASED ROE DEER FAWN DETECTION SYSTEM
}

\author{
Martin Israel \\ Remote Sensing Technology Institute, Experimental Methods \\ German Aerospace Center \\ Oberpfaffenhofen, 82234 Wessling, Germany \\ martin.israel@dlr.de \\ http://www.dlr.de/caf
}

KEY WORDS: UAV, Thermal Imaging, Animal Rescue, Animal Welfare, Remote Sensing, Detection System, Agriculture, Octocopter

\begin{abstract}
:
This paper presents a UAV based remote sensing system for the detection of fawns in the meadows. There is a high demand because during pasture mowing many wild animals, especially roe deer fawns are killed by mowing machines. The system was tested in several real situations especially with differing weather and iluminating conditions. Its primary sensor is a lightweight thermal infrared camera. The images are captured onboard of the flight system and also transmitted as analog video stream to the ground station, where the user can follow the camera live stream on a monitor for manual animal detection. Beside a high detection rate a fast workflow is another very important objective for this application. Therefore a waypoint planning software was developed that accelerates the workflow. At adequate illuminating and weather conditions the presented UAV-based fawn detection via thermal imaging is a comfortable, fast and reliable method.
\end{abstract}

\section{INTRODUCTION}

Beside economic benefits the progressive industrialization of agriculture involves also problems. Growing working width and higher velocities of the harvesters lead to an increasing danger for wild animals living in the farmland. Roe deer (capreolus capreolus) fawns are classic hiders (Espmark, 1969) and therefore frequent victims of agricultural accidents. They lie hidden in seclusion for most of the time during their first two months of life (Jarnemo, 2002). The cultivated pasture farmland is a favored bedsite for the neonate roe deer, because it screens them from the predators' view. $96 \%$ of the fawns are born between May and June (Rieck, 1955), the time of pasture mowing. Because of their innate instinct to remain motionless on the ground, they are often overlooked by farmers. About $25 \%$ of the newborn deers are killed during pasture mowing (Jarnemo, 2002). Beside animal welfare there is another reason for the importance to avoid these accidents. If the carcasses get undetected in the silage, bacteria produce a mortally poison (botulinum toxin) that causes health hazard in the fodder for cows, resulting in critical economic loss for the farmer. Since the beginning of the agricultural industrialization people try to prevent these accidents. Within the Game Guard project several systems are developed (Israel et al., 2010).

There are two approaches to do that: shooing the animals by scaring devices or detecting and carrying them away. The first one follows the idea, that the does will choose another area for fawn bed site, and they will remove their fawns already hiding in the field (Jarnemo, 2002). An authoritative study that evidences success with this method does not exist and in several tests with plastic bags as scaring device fawns lay in the same region two days after mounting the devices. In some cases fawns actually were found directly beside the scaring device. We are conviced, that this method does not work properly. The system described in this contribution follows the second approach (to detect the animals and carry them away).

Actually it is not really easy to find a fawn in the meadow. In the visible spectrum the fawns are very good camouflaged. They have no scent in the first days of life and they keep silent and motionless on the ground. The evolution has done a very good job to adapt them to the surrounding, so that for predators and people it's hard to find them.

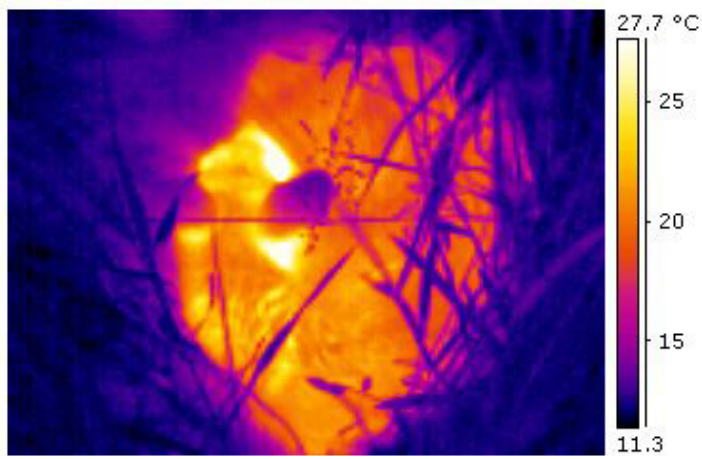

Figure 1: thermal image of a fawn

In the thermal infrared spectrum fawns are not adapted that perfectly. The coat of the newborn deer is barely insulating. Therefore it is detectable with a thermal camera if the surrounding is cold enough (figure 1). But even with a hand-held thermal camera a walking person hardly finds a fawn in the meadow. The grass of the meadow in which fawns prefer to lie is that dense that the thermal radiation does not penetrate it.

This contribution presents a fawn detection system consisting of a thermal camera that is mounted on a UAV. Flying some ten meters above the ground with the view directed vertically down enables to cover a much wider area than with any other tested system until now. 


\section{MATERIAL AND METHODS}

\subsection{Geometric considerations}

This section treats with the geometric condition that enables the detection of fawns with a camera. The trigonometrical equation

$$
\tan (\alpha)=\frac{f}{h}=\frac{\bar{f}}{\bar{h}}
$$

shows that the maximum angle deviation $\alpha$ between the viewing direction and the nadir is ca. $17^{\circ}$ in order to detect a fawn with a size $f$ of $30 \mathrm{~cm}$ in a meadow with an average grass height $h$ of $1 \mathrm{~m}$ due to visual occlusion (figure 2 ). $\bar{f}$ is half of the swath and $\bar{h}$ is the distance between the camera and the ground.

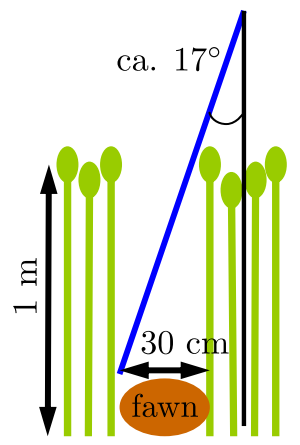

Figure 2: geometric boundary conditions

So for this application the camera works best with a $34^{\circ}$ field of view angle (the double of $\alpha$ ) for optics pointing down vertically. Then the grass can only occlude a fawn completely at the edges of the image independently from the distance of the camera to the ground. The higher the distance the larger is the covered area with one picture. But with increasing distance the ability to detect a fawn decreases due to less detailed information. Equation 1 can also be used to calculate the swath of the camera with a given field of view (FOV) angle and distance to the ground. The size of one pixel on the ground is given by

$$
s=\frac{2 \bar{f}}{p}
$$

where $p$ is the cameras amount of pixels. Solving equation 2 for $\bar{f}$ and substituting $\bar{f}$ in equation 1 results in equation 3 , which shows the correlation between the flight altitude $\bar{h}$ and the size $s$ that represents one pixel on the ground without regarding aberrations.

$$
\bar{h}=\frac{p s}{2 \tan (\alpha)}
$$

When the mean body temperature of the fawn holds all the neccesary information to classify the fawn in the meadow, then a pixel resolution corresponding to the size of a fawn would be sufficient. According to the Nyquist-Shannon sampling theorem (equation 4) the sampling frequency $f_{\text {samp }}$ has to be more than twice of the highest information relevant frequency $f_{\max }$ to avoid information loss (Shannon, 1949). That means: size of a fawn is about $30 \mathrm{~cm}$, therefore the pixel size on the ground has to be less than $15 \mathrm{~cm}$.

$$
f_{\text {samp }}>2 f_{\max }
$$

Considering all this the commercially available thermal camera core Tau640 from FLIR with $640 \times 512$ pixels and optics with $32^{\circ} \times 26^{\circ}$ FOV was selected. Using equation 3 the camera with this specification can theoretically detect a fawn in the meadow from a maximum altitude of $166 \mathrm{~m}$. Flying at a lower altitude raises the detection certainty.

\subsection{System description}

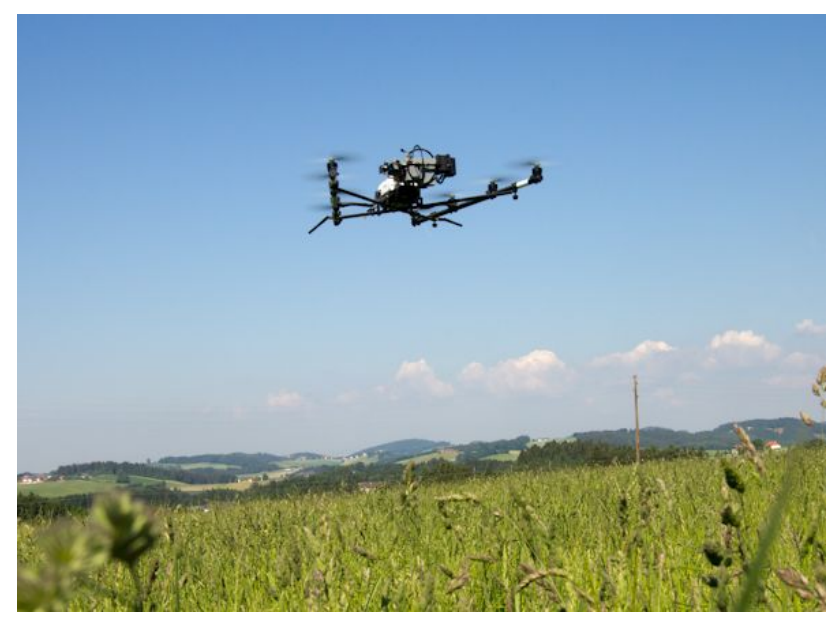

Figure 3: flying Game Guard in action

We mounted our thermal camera on the micro air vehicle (MAV) Falcon-8 from Ascending Technologies GmbH (figure 3). A rotorcraft like this Octocopter is able to hover due to its GPS-, pressure- and magnetic field sensor. This allows capturing georeferenced high quality images of the scene. In our application we scan the whole area by flying line by line capturing images in a stop-and-go mode at equidistantly spaced waypoints. The red line in figure 4 shows a schematic flight path. The gray quadrangles represent the observed area on the ground. There is an overlap in both directions to guarantee full coverage. The blue circle represents the MAV. Flying at high altitude means the camera covers a wider swath. A shorter flight path is needed to cover the whole area than by flying at lower altitude. Moreover less waypoints are necessary and that saves time, because settling the waypoint, stoping for capturing and accelerating to reach the next waypoint takes a while. The disadvantage in flying at high altitude is the lower resolution. Identifying the fawns is therefore more difficult. Detecting hot spots at high altitude and identify them at lower altitude is a promising approach.

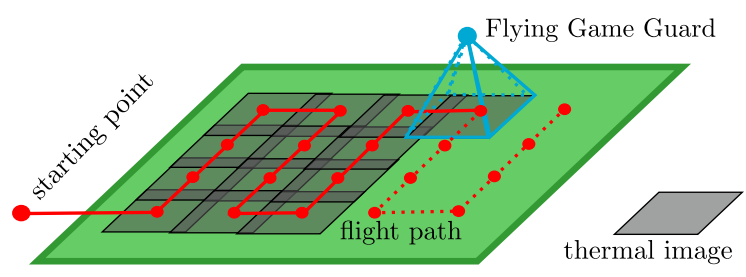

Figure 4: coverage of the field

Figure 5 shows a simplified diagram of the system architecture. The analog videostream of the thermal camera 
is sent through a $5.8 \mathrm{GHz}$ radio link to a monitor at the ground station, where the pilot can follow the view of the onboard camera. In parallel the images are captured onboard via a frame grabber by a miniature computer on module (Gumstix) with a Linux operating system. The capturing software on the Gumstix is triggered by a Universal Asynchronous Receiver Transmitter (UART) package sent by the Falcon- 8 while it reaches the next waypoint. The UART package contains the current GPS position, flight altitude and camera inclination angle. This information and the thermal image are stored for each waypoint on a microSD card. The pattern recognition algorithm prospectively sends its results via the UART interface and the radio link down to the ground station to inform the pilot and give him a decission support.

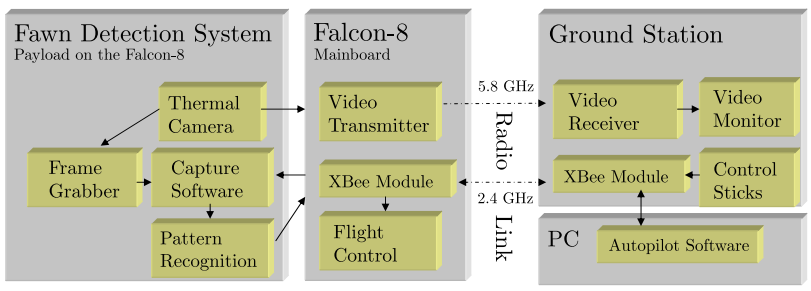

Figure 5: system architecture diagram

At many other UAV-based pattern recognition projects the images were sent directly to the ground station, where a pattern recognition algorithm processes the images. Our system has the pattern recognition machine onboard. This prevents difficulties with interferences of the video transmission via radio link, but involves less cpu power for the pattern recognition due to the limited payload. Additionally we have mounted a visual camera on the payload. Switching between the two video sources that are displayed on the monitor can be done remotely at the ground station. The pattern recognition algorithm does not yet work on the gumstix, therefore currently the user has to detect the fawn by following the video stream on the monitor (Israel and Evers, 2011).

\subsection{Workflow}

Once the grass has grown and the weather forecast predicts sunny weather, all the farmers are mowing at the same time, because before making silage the cut grass has to dry some hours. Therefore searching fawns in the time of pasture mowing is a really time critical task. The less time it takes to detect a fawn, the more fawns can be saved. For fawn detection and rescueing with the flying Game Guard at least two persons are necessary. One person acts as pilot: He has to keep an eye on the flight system and has to bring it manually save to the ground in emergency cases. Another person (at best the tenant of the local hunt) is required to walk to the fawn and carry it away. A third person might be useful to operate the pc, communicate with the hunter and keep track of the thermal videostream on the monitor.

The workflow is shown in figure 6 . It consists of the following processes: waypoint planning and preparation is a task that has to be done before the flight. Often the flight path can be defined at the earliest while standing at the edge of the meadow, because sometimes a good launch position can be assessed only on site. Hot spot detection and placemarking them happens while flying the search mission at a

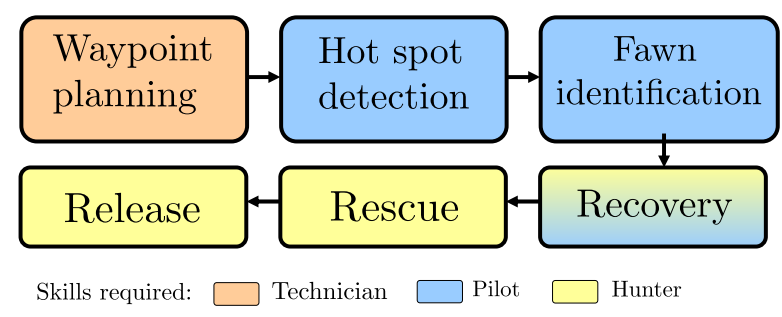

Figure 6: Workflow of the fawn rescueing process

high altitude (30 to $50 \mathrm{~m}$ ). Fawn identification is the next step. The pilot manually positions the copter in a lower altitude above the placemarked hot spot and tries to identify it by observing the thermal and visual videostream on the monitor. The GPS position of an identified fawn is noted in a hand-held GPS device. After the flight this tool guides the hunter to the fawn's position. If the hunter does not find the fawn with his GPS device, then an assisted recovery with the octocopter will yield the desired result: The pilot can guide the hunter by watching the scene from above. Rescueing the fawn is a process the hunter can do by himself. He picks it up with gloves or hassocks so that the fawn does not acquire the odor of the human. Then he carries the fawn away of the meadow and pens it in a cage to prevent it from refraining to the field. After mowing the field the hunter can release the fawn.

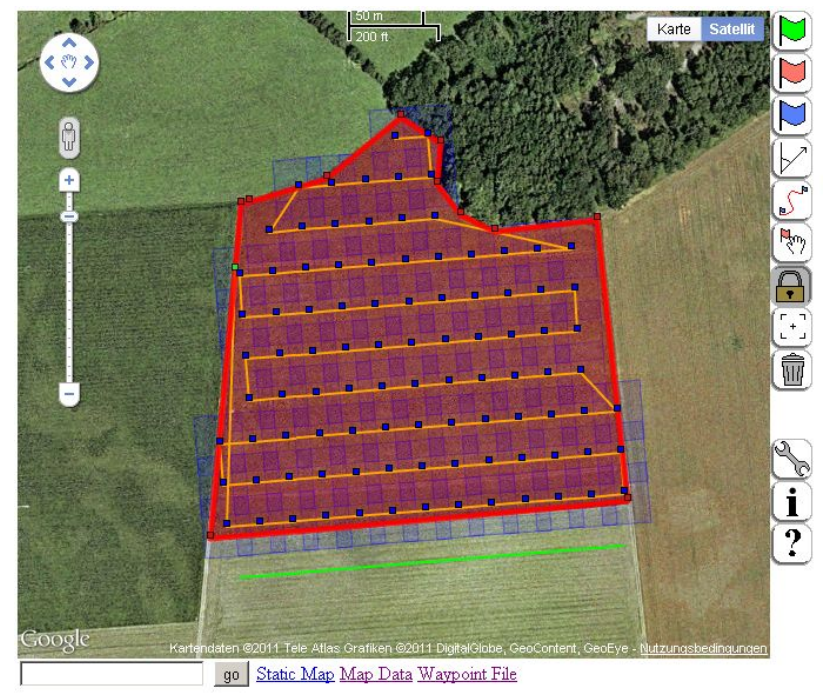

Figure 7: Screen shot of the Waypoint Editor

The waypoint planning based on the standard methods the manufacturer provides proved to be exhausting and very time consuming. Therefore we developed the Waypoint Editor, a web application based on the Google Maps Application Programming Interface (API). This application requires an internet connection. By means of a satellite image it's easy for the user to mark the corners of the field and the launch position of the octocopter. The application calculates then an optimized flight path with a predefined distance beween each waypoint. Even the flight path for a complex area can be constructed fast. An image overlap, the copter heading, flight altitude and further parameters can be configured. Figure 7 shows a screen shot of the Waypoint Editor. The red polygon is the desired scan area which the user marked out. Adjusting the corners of the 
scan area causes an automatic adaption of the waypoints. The software shows the calculated flight path as a yellow line. Every waypoint is marked by a blue dot surrounded by the field of view on the ground which is represented by a transparent blue rectangle.

The flight altitude of the Falcon- 8 is measured by a preasure sensor. Therefore statements on the flight altitude are referenced relatively to the starting position. Flying in a line on the same altitude above hills causes a variation of the distance to ground. In worst case this variation can lead to a gap in the image coverage. To avoid this the Waypoint Editor adapts the flight altitude of each waypoint based on an elevation map the Google Maps Elavation API provides. The resulting waypoint list can be imported into the autopilot software.

\subsection{Thermal camera}

To reach a high sensitivity each pixel of the thermal detector (focal plane array) provides a 14-bit signal (>15000 grey levels). The human visual system can distinguish only approx. 128 levels of grey (7 bit) in an image. In addition many analog and digital video interfaces require 8 bit values which effectively limits the dynamic range to 256 levels of grey (Högasten and Lindner, 2009). Hence the raw image with 14-bit has to be compressed. Most of the common thermal cameras provide an automatic gain control (AGC) that calculates a high contrast for the scene. FLIR offers some parameters (Plateau Value, ITT Mean and Max Gain) to influence the results of their automatic gain control.
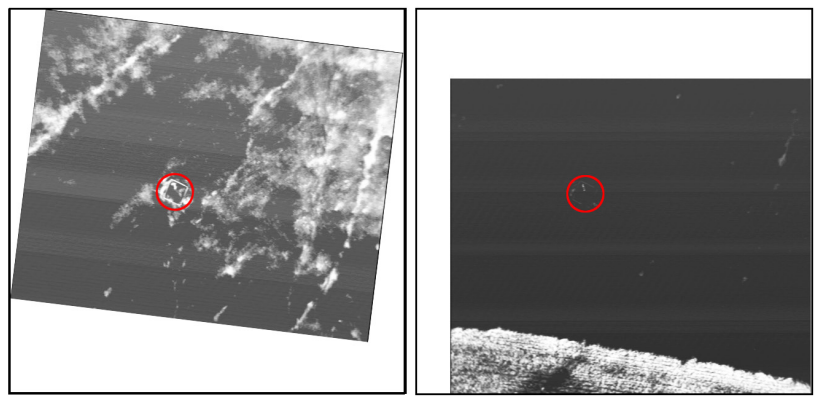

Figure 8: variation of the contrast with the Automatic Gain Control. Flight altitude: $30 \mathrm{~m}$

Figure 8 demonstrates the reduction of contrast of a scene with standard AGC configuration when a warm area comes into view (right image). The red circle marks the same fawn surrounded by a fence in both pictures. It often happens that there is a street at the edge of a field, which is warmer than the vegetation. Therefore we decided to change the AGC configuration and use the linear histogram equalization instead. This proved to be by far more robust for these situations compared to standard plateau histogram equalization.

Some hot spot examples are shown in figure 9. These images are captured during field campaigns in May 2011. The small images on the left are enhanced $64 \times 64$ pixel sized cut-outs of captured thermal images. The upper images are no fawns (a: warmed ground spot, b: anthill, c: hay bale) and the lower images are fawns (on image $d$ are even two fawns). Image $\mathrm{a}, \mathrm{b}, \mathrm{d}$ and e are taken from an altitude of $30 \mathrm{~m}$, the rest from $50 \mathrm{~m}$. Image $\mathrm{f}$ is a cut-out of the full-sized thermal image g. At a flight altitude of $50 \mathrm{~m}$ a fawn is represented by just 40 of 327680 pixels.

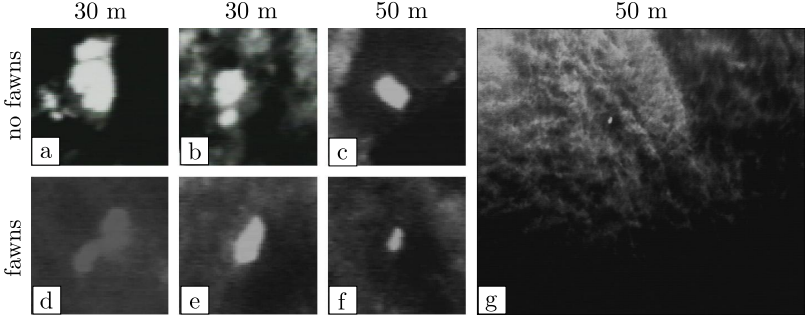

Figure 9: thermal image cut-outs of typical hot spots at a flight altitude of 30 and $50 \mathrm{~m}$

\section{RESULTS AND DISCUSSION}

In May and June 2011 we arranged field campaigns on 15 days. 70.77 hectare (ha) were scanned at different time of day, illuminating and weather conditions. 44.2 ha were scaned at 22 flights at a flight altitude of $50 \mathrm{~m}$ and 26.6 ha at 28 flights at $30 \mathrm{~m}$. The average time of flight to scan one hectare at an altitude of $50 \mathrm{~m}$ was $3: 47$ minutes. At an altitude of $30 \mathrm{~m}$ it was 8:48 minutes. Launching and landing took about one minute.

Flying higher than $50 \mathrm{~m}$ sometimes led to a fawn miss even at best illuminating and weather conditions. At worse conditions flying at $30 \mathrm{~m}$ often could improve the fawn recognition. Flying lower than that is not practical due to the limited search performance.

At all flights the image overlap in both directions was $30 \%$, which led to a complete coverage of the scan area at all missions. For missions at flat areas a lower value could suffice. During the campaigns we found with this system 14 fawns, three adult deers, five rabbits, one fox and some smaller animals. Most of them were found between 2nd and 7 th of June. The illuminating conditions at these days were optimal. At the field campaigns between 10th to 26th the illuminating conditions were disadvantageous. Just one fawn could be found.

This year the vegetation height was very low due to a dry and very hot period in April. Thus, many farmers mowed their meadows in May at a vegetation height of less than $40 \mathrm{~cm}$. Because fawns prefer to lie in meadows with higher vegetation this year less fawns are killed during mowing.

\section{CONCLUSIONS AND FUTURE WORK}

This contribution depicts the problem of mortally injured roe deer fawns by mowing machines and demonstrates a technical sophisticated "detection and carry away" solution to avoid these accidents. The architecture of the fawn detection system consisting of a thermal camera mounted on a Micro Air Vehicle was described. Also the workflow of the whole fawn rescueing process was presented. The special characteristics of the fawn detection by thermal imaging was shown. The field campaigns enabled to evaluate the conditions of a reliable detection of fawns in the meadows by thermal imaging. It has shown that the manual detection is fast and reliable in absence of the sun. Future work will concentrate on implementing the automatic pattern recognition algorithm on the payload and the sensor data fusion between the thermal and the visual camera to extend the detection rate during sunshine. Moreover it is planed to optimize the usability and the operating time of the whole workflow. 


\section{ACKNOWLEDGEMENTS}

The work described is part of the Game Guard Project which is funded by the Bundesministerium für Bildung und Forschung (BMBF). The author would like to thank Dr. Moser from Bad Zell in Austria for his helpful support and expertise in the field of fawn rescueing. Thanks also go to Daniel Gurdan (CEO from Ascending Technologies $\mathrm{GmbH}$ ) for his quick support and especially for the custom adaptions of the flight system.

\section{REFERENCES}

Espmark, Y., 1969. Mother-young relations and development of behaviour in roe deer: Capreolus capreolus 1 .

Högasten, N. and Lindner, R., 2009. Digital detail enhancement (dde). Technical report, Flir.

Israel, M. and Evers, S., 2011. Pattern recognition for fawn detection in thermal images. In: 17 . Workshop ComputerBildanalyse in der Landwirtschaft - Computerized Image Analysis in Agriculture.

Israel, M., Schlagenhauf, G., Fackelmeier, A. and Haschberger, P., 2010. Study on wildlife detection during pasture mowing. In: 68. Internationale Tagung LAND.TECHNIK, Braunschweig.

Jarnemo, A., 2002. Roe deer capreolus capreolus fawns and mowing-mortality rates and countermeasures. Wildlife Biology 8(3), pp. 211-218.

Rieck, W., 1955. Die Setzzeit bei Reh-, Rot-und Damwild in Mitteleuropa. Zeitschrift für Jagdwissenschaft 1(2), pp. 69-75.

Shannon, C., 1949. Communication in the presence of noise. Proceedings of the IRE 37(1), pp. 10-21. 